\title{
PADRÃO DE ATIVIDADE FÍSICA EM MULHERES COM EXCESSO DE PESO
}

\author{
STANDARD OF PHYSICAL ACTIVITY IN WOMEN \\ WITH EXCESSIVE WEIGHT
}

\section{ESTÁNDAR DE ACTIVIDAD FÍSICA EN MUJERES CON EXCESO DE PESO}

\author{
Taise Santos do Nascimento ${ }^{1}$ \\ Fernanda Carneiro Mussi ${ }^{2}$ \\ Cátia Suely Palmeira ${ }^{3}$ \\ Francisco José Godim Pitanga ${ }^{4}$ \\ Melissa Almeida Santos Paim ${ }^{5}$
}

Como citar este artigo: Nascimento TS, Mussi FC, Palmeira CS, Pitanga FJG, Paim MAS. Padrão de atividade física em mulheres com excesso de peso. Rev baiana enferm. 2018;32:e25738.

\begin{abstract}
Objetivo: descrever o nível de atividade física de mulheres com excesso de peso. Método: estudo transversal, descritivo. Cento e quarenta e três mulheres foram entrevistadas, utilizando-se o International Physical Activity Questionnaire, e submetidas a avaliação antropométrica, no período de outubro de 2015 a julho de 2016. Os dados foram analisados em frequências relativas e absolutas, médias e desvio padrão (DP). Resultados: idade média foi 50,66 anos ( $\mathrm{DP}=11,55)$. Predominaram mulheres negras, sem companheiro, com até o segundo grau, em atividade laboral e baixa renda. Maior proporção era obesa $(82,5 \%)$, com circunferência da cintura não recomendada ( $\geq 80 \mathrm{~cm}$ ). Referente ao nível de atividade física global, 65,4\% eram ativas/muito ativas. Considerando o nível de atividade física por domínio, 64,4\% eram insuficientemente ativas/sedentárias no trabalho, 78,1\% no deslocamento e $85,2 \%$ no tempo livre, mas $54,2 \%$ eram ativas/muito ativas nas atividades domésticas. Conclusão: o nível de atividade física de mulheres com excesso de peso foi baixo na maioria dos domínios, exceto no domicílio, sendo necessário encontrar estratégias para otimizar a prática de atividade física sobretudo no lazer.
\end{abstract}

Descritores: Atividade Motora. Mulheres. Obesidade.

Objective: to describe the level of physical activity of overweight women. Method: this is a cross-sectional and descriptive study. One hundred and forty-three women were interviewed, using the International Physical Activity Questionnaire, and were submitted to anthropometric evaluation, from October 2015 to July 2016. Data were analyzed in relative and absolute frequencies, means and standard deviation (SD). Results: mean age was 50.66 years (SD=11.55). There was a predominance of black women, with no partner, with education up to bigh school degree, working, and with low income. The majority was obese (82.5\%), with waist circumference not recommended ( $\geq 80 \mathrm{~cm})$. Regarding the level of global physical activity, 65.4\% were active/very active. Considering the level of physical activity per domain, $64.4 \%$ were insufficiently active/sedentary at work, $78.1 \%$ were on the move and $85.2 \%$ were in free time, but $54.2 \%$ were active/very active in domestic activities. Conclusion: the level of physical activity of overweight women was low

\footnotetext{
Enfermeira. Mestre em Enfermagem. Salvador, Bahia, Brasil.

Enfermeira. Doutora em Enfermagem. Professora Associada IV da Escola de Enfermagem da Universidade Federal da Bahia. Salvador, Bahia, Brasil. femussi@uol.com.br

Enfermeira. Doutora em Enfermagem. Professora da Faculdade Baiana de Medicina e Saúde Pública. Salvador, Bahia, Brasil.

Educador Físico. Doutor em Saúde Coletiva. Professor Associado da Universidade Federal da Bahia. Salvador, Bahia, Brasil.

Enfermeira. Membro do Grupo Interdisciplinar sobre o Cuidado à Saúde Cardiovascular. Salvador, Bahia, Brasil.
} 
in most domains, except at home, and it is necessary to find strategies to optimize the practice of physical activity, especially in leisure.

Descriptors: Motor Activity. Women. Obesity.

Objetivo: describir el nivel de actividad física de mujeres con exceso de peso. Método: estudio transversal, descriptivo. Ciento cuarenta y tres mujeres fueron entrevistadas, utilizándose el International Physical Activity Questionnaire, y sometidas a evaluación antropométrica, de octubre de 2015 a julio de 2016. Datos analizados en frecuencias relativas y absolutas, medias y desviaciones estándar (DE). Resultados: edad media de 50,66 años $(D E=11,55)$. Predominaron mujeres negras, sin compañero, con hasta el segundo grado, en actividad laboral y baja renta. Mayor proporción era obesa $(82,5 \%)$, con circunferencia de la cintura no recomendada $(\geq 80 \mathrm{~cm})$. En cuanto al nivel de actividad fisica global, 65,4\% eran activas/muy activas. En cuanto al nivel de actividad fisica por dominio, 64,4\% eran insuficientemente activas/sedentarias en el trabajo, 78,1\% en el desplazamiento y 85,2\% en el tiempo libre, pero $54,2 \%$ eran activas/muy activas en las actividades domésticas. Conclusión: el nivel de actividad física de mujeres con exceso de peso fue bajo en la mayoría de los dominios, excepto en el domicilio, siendo necesario encontrar estrategias para optimizar la práctica de actividad física sobre todo en el ocio.

Descriptores: Actividad Motora. Mujeres. Obesidad.

\section{Introdução}

A transição demográfica, epidemiológica e nutricional ocorrida nas últimas décadas tem favorecido o aumento da prevalência de doenças crônicas não transmissíveis (DCNT), dentre elas a obesidade $^{\mathrm{O}}$, a qual configura-se como um problema de saúde pública mundial, com prevalência crescente nos últimos anos, atingindo ambos os sexos e diferentes faixas etárias. Estima-se que mais da metade da população brasileira apresente excesso de peso $(56,2 \%)$, isto é, $82 \mathrm{mi}-$ lhões de pessoas apresentam Índice de Massa Corporal (IMC) igual ou maior que $25 \mathrm{~kg} / \mathrm{m}^{2}$, sendo maior no sexo masculino $(56,5 \%)$ do que no sexo feminino (49,1\%). Em relação à obesidade (IMC> $29,9 \mathrm{~kg} / \mathrm{m}^{2}$ ), observa-se maior prevalência no sexo feminino $(18,2 \%)$ do que no masculino $(17,6 \%)^{(2)}$

A etiologia da obesidade é complexa e multifatorial, tendo, como determinantes, fatores históricos, ecológicos, econômicos, sociais, psicológicos, culturais e políticos, sendo a sua prevenção e controle um desafio no cenário mundial. O excesso de peso destaca-se como um fator de risco associado ao aumento da pressão arterial sistêmica, intolerância à glicose e dislipidemia, diabetes mellitus tipo 2 e doenças cardiovasculares ${ }^{\mathrm{O}}$.
Os benefícios da prática regular da atividade física para redução e controle do peso corporal são bem conhecidos ${ }^{(3)}$, trazendo contribuições fisiológicas, como aumento da taxa metabólica basal, melhor controle glicêmico e lipídico e qualidade do sono ${ }^{(4)}$, além da redução ou prevenção de algumas doenças como osteoporose e desvios de postura. Na esfera psicológica observa-se redução dos níveis de ansiedade, estresse e depressão ${ }^{(5)}$. Na dimensão social, os indivíduos podem obter melhor integração social e cultural $^{(6)}$.

Apesar desses benefícios, o século vinte foi marcado pelo crescimento da inatividade física. Prevalências superiores para inatividade física têm sido encontradas em mulheres, relacionando-se ao engajamento com questões familiares e papéis relacionados ao de esposa, mãe e cuidadora, bem como à falta de suporte social, de segurança pública e aspectos climáticos ${ }^{(7-9)}$.

A análise da literatura evidenciou que a maioria dos estudos relacionados à caracterização do padrão de atividade física de mulheres com excesso de peso provém de países desenvolvidos $^{(5,7-8)}$ e conhecê-lo no contingente brasileiro evidencia a vulnerabilidade das mulheres ao adoecimento e orienta para a criação e 
implementação de programas efetivos para minimizar esse problema de saúde pública.

Com base no exposto, o presente trabalho teve como objetivo descrever o nível de atividade física de mulheres com excesso de peso.

\section{Método}

Trata-se de um estudo descritivo, recorte de um projeto matriz denominado "Monitoramento Remoto de Enfermagem de Mulheres com Excesso de Peso", desenvolvido em um serviço de referência para obesidade localizado na cidade de Salvador, Bahia, Brasil, no qual uma equipe multidisciplinar atende usuários do Sistema Único de Saúde (SUS). Atualmente cerca de 300 pessoas com excesso de peso são atendidas, sendo a maioria mulheres (91\%), razão pela qual foram selecionadas como participantes do estudo.

A coleta de dados ocorreu no período de outubro de 2015 a julho de 2016. Mediante consulta aos prontuários, identificou-se que 174 mulheres com excesso de peso atenderam aos critérios de elegibilidade definidos para o projeto matriz. Dessas, 143 aceitaram participar. Foram critérios de inclusão: ter excesso de peso (IMC $\geq 25 \mathrm{~kg} / \mathrm{m}^{2}$ ), idade superior a 18 anos, comparecido, no mínimo, a uma consulta nos últimos 12 meses e telefone fixo e/ou celular. Foram critérios de exclusão: ausência de condições físicas (amputações) para realização das medidas de peso e altura, confusão mental e condições cognitivas que impedissem responder aos instrumentos da pesquisa e ler mensagens escritas, distúrbios psiquiátricos já diagnosticados, em uso de drogas para perda de peso e submetidas à cirurgia bariátrica.

Na coleta de dados foi utilizado um instrumento para caracterização sociodemográfica, com questões fechadas sobre idade, raça/cor autodeclarada, estado civil, escolaridade, situação laboral, renda familiar mensal, com quem mora, presença de empregada doméstica e tempo de acompanhamento no serviço.

Para a avaliação do padrão de atividade física foi utilizado o International Physical Activity
Questionnaire(IPAQ) versão longa, devidamente validado $^{(10)}$. O instrumento contém perguntas relacionadas à frequência (dias por semana), duração (tempo por dia) e intensidade (leve, moderada e vigorosa) da atividade física desenvolvida no trabalho, no deslocamento, nas atividades domésticas e no tempo livre. Este instrumento permite a classificação de acordo com o escore obtido em quatro níveis: indivíduos muito ativos, ativos, insuficientemente ativos e sedentários. Os indivíduos foram classificados como muito ativos quando cumpriram as recomendações de realização de atividade física vigorosa: $\geq 5$ dias/ sem e $\geq 30$ minutos por sessão ou $\geq 3$ dias/sem e $\geq 20$ minutos por sessão + moderada ou caminhada: $\geq 5$ dias/sem e $\geq 30$ minutos por sessão. Indivíduos ativos cumpriram a recomendação de atividade física vigorosa: $\geq 3$ dias/sem e $\geq$ 20 minutos por sessão; ou moderada ou caminhada: $\geq 5$ dias/sem e $\geq 30$ minutos por sessão; ou qualquer atividade somada: $\geq 5$ dias/sem e $\geq$ 150 minutos/sem (caminhada + moderada + vigorosa). Insuficientemente ativos foram aqueles que realizaram atividade física, porém de forma insuficiente para serem classificados como ativos, pois não cumpriram as recomendações quanto à frequência ou duração. Foi classificado como sedentário quem não realizou nenhuma atividade física por pelo menos 10 minutos contínuos durante a semana.

Para verificação das medidas antropométricas, as participantes estavam descalças, usando roupas leves com tecido Kami, sem adereços. O peso (quilogramas) foi verificado usando uma balança digital scale, model TEC 30 da marca Techline ${ }^{\circledR}$, com variação de $0,1 \mathrm{~kg}$. A altura (metros) foi definida usando-se estadiômetro portátil (graduado a cada $0,5 \mathrm{~cm}$ ).

Para aferição do peso, foi solicitado às mulheres que ficassem de pé, braços ao longo do corpo e olhar fixo no horizonte. Após aferição do peso, permaneceram de pé para verificação da altura, com cabeça e dorso encostados na régua do estadiômetro, abaixo da haste horizontal, mantendo braços esticados, ombros, omoplatas, nádegas, calcanhares encostados na parede e pés no chão. Em seguida, foi-lhes 
solicitado que olhassem para frente, inspirassem fundo e prendessem o ar.

A verificação dessas variáveis (peso e altura) foi usada para determinar o índice de massa corpórea (IMC), cuja fórmula é a razão entre o peso em quilogramas e o quadrado da altura em metros $\left(\mathrm{IMC}=\mathrm{peso} /\right.$ altura $\left.^{2}\right)$. As mulheres foram classificadas segundo critérios da Organização Mundial de Saúde ${ }^{(11)}$ da seguinte forma: sobrepeso (IMC 25 a 29,9 kg/m²); obesidade Grau I (30 a $34,9 \mathrm{~kg} / \mathrm{m}^{2}$ ); Obesidade Grau II (35 a $39,9 \mathrm{~kg} / \mathrm{m}^{2}$ ) e obesidade Grau III (IMC $\geq 40 \mathrm{~kg} / \mathrm{m}^{2}$ ).

A circunferência da cintura foi aferida no ponto médio entre a última costela e a crista ilíaca ântero-superior na linha axilar média, lado direito. As participantes ficaram de pé, abdômen relaxado, respirando normalmente, braços estendidos, peso igualmente distribuído entre as pernas, pés próximos e paralelos. A região da cintura estava desprovida de roupa. A fita métrica utilizada foi flexível, inelástica com precisão de $0,1 \mathrm{~cm}$.

O parâmetro utilizado para avaliação e classificação para CC foi o da International Diabetes Federation (IDF), que propõe ponto de corte que difere entre etnias, sendo adotado aquele designado para o grupo étnico centro sul e americanos ( $\leq 80 \mathrm{~cm}$ para mulheres $)^{(12)}$.

Para circunferência do quadril, o examinador permaneceu na posição sentada, em frente à participante, a qual estava de costas para um espelho, e realizou-se a medida com trena métrica circundando a protusão máxima dos músculos do quadril. A relação cintura-quadril foi determinada pela divisão da circunferência da cintura $(\mathrm{cm})$ pela circunferência do quadril $(\mathrm{cm})$, sendo considerada elevada se $\geq 0,85 \mathrm{~cm}^{(13)}$.
O projeto de pesquisa foi aprovado por Comitê de Ética em Pesquisa (CEP), número do processo 1.152 .259 e atendeu à Resolução n. 466/2012 do Conselho Nacional de Saúde. As mulheres elegíveis receberam informações acerca dos objetivos e procedimentos da pesquisa, riscos e benefícios, assegurando-se o direito de desistência e sigilo das informações. Assinaram o Termo de Consentimento Livre e Esclarecido (TCLE) em duas vias, sendo uma via para a participante e outra para as pesquisadoras.

Os dados constituíram um banco no programa Statistical Package for Social Science (IBM SPSS versão 18.0), onde foram processados e analisados. As variáveis categóricas foram analisadas em frequências absolutas e relativas e as contínuas, em médias e desvio padrão.

\section{Resultados}

Quanto às características sociodemográficas, observou-se média de idade de 50,66 anos (DP=11,59), idade mínima de 21 e máxima de 81 anos. Predominaram autodeclaradas da raça/ cor negra $(93,7 \%)$, com ensino fundamental completo ou incompleto $(62,4 \%)$ e união estável/casadas (55,9\%). A maioria vivia na companhia de alguém $(86,0 \%)$ e não tinha empregada doméstica $(95,1 \%)$ e apresentava situação laboral atual remunerada (51,05\%) por serem autônomas, aposentadas com atividade ou possuírem vínculo empregatício. A maior proporção trabalhava menos de 8 horas por dia $(65,7 \%)$ e tinha renda familiar de 1 a 3 salários mínimos $(39,1 \%)$, da qual dependiam em média 3 pessoas $(\mathrm{DP}=1,32)$. (Tabela 1).

Tabela 1 - Características sociodemográficas das mulheres com excesso de peso. Salvador, Bahia, Brasil - $2016(\mathrm{~N}=143)$

(continua)

\begin{tabular}{lc}
\hline Características Sociodemográficas & n (\%) \\
\hline Idade & $25(17,4)$ \\
18 a 39 anos & $90(62,9)$ \\
40 a 59 anos & $28(19,5)$ \\
$\geq 60$ anos & \\
Raça/Cor & $9(6,3)$ \\
Branca & $134(93,7)$ \\
Negra (parda e preta) &
\end{tabular}


Tabela 1 - Características sociodemográficas das mulheres com excesso de peso. Salvador, Bahia, Brasil - $2016(\mathrm{~N}=143)$ (conclusão)

\begin{tabular}{lc}
\hline Características Sociodemográficas & n (\%) \\
\hline Situação conjugal & $80(55,9)$ \\
$\quad$ Casada / com companheiro & $63(44,0)$ \\
$\quad$ Sem companheiro & \\
Escolaridade & $48(33,5)$ \\
$\quad$ Até Ensino Fundamental Incompleto & $95(66,4)$ \\
$\quad$ Ensino médio incompleto, completo e superior & $123(86)$ \\
Vive na companhia de alguém & $75(51,05)$ \\
Situação laboral remunerada & \\
Horas de trabalho/dia & $46(65,7)$ \\
$\quad \leq 8$ h/dia & $24(34,3)$ \\
$>8$ h/dia & \\
Renda Familiar Mensal & $37(25,8)$ \\
$\geq 3$ salários mínimos & $56(39,1)$ \\
1 a 3 salários mínimos & $50(34,7)$ \\
$\leq 1$ salário mínimo & $136(95,1)$ \\
Não tem empregada doméstica & $44(30,8)$ \\
Pessoas dependentes no domicílio &
\end{tabular}

Fonte: Elaboração própria.

A média do IMC foi $36,29 \mathrm{~kg} / \mathrm{m}^{2}$ (DP=6,23), valor mínimo de $25,0 \mathrm{~kg} / \mathrm{m}^{2}$ e máximo de 50,4 $\mathrm{kg} / \mathrm{m}^{2}$. Houve predomínio de mulheres obesas (82,5\%), estando 31,5\% com obesidade grau III, $29,37 \%$ com obesidade grau I e 21,7\% com obesidade grau II. Apresentaram sobrepeso 17,5\%.

Quanto à circunferência da cintura, 98,6\% das mulheres tinham CC $\geq 80 \mathrm{~cm}$. A média da CC foi de 105,28 cm (DP=11,97), valor mínimo de $74 \mathrm{~cm}$ e máximo de $133 \mathrm{~cm}$. Para a razão cintura quadril, avaliada em 141 mulheres, observou-se que 69,9\% estavam com valores aumentados $(>0,85 \mathrm{~cm})$, com média de $0,89 \mathrm{~cm}$, valor mínimo de 0,56 cm e máximo de 1,23 m ( $\mathrm{DP}=0,08)$.

Quanto ao nível de atividade global avaliada pelo IPAQ (Tabela 2), isto é, referente à avaliação dos quatro domínios em conjunto (trabalho, deslocamento, ambiente doméstico e tempo livre), $65,5 \%$ das mulheres foram consideradas ativas/ muito ativas, pois atingiram a recomendação de prática de atividade física de $\geq 150 \mathrm{~min} /$ semana; $34,51 \%$ foram classificadas como irregularmente ativas/sedentárias, isto é, não atingiram a recomendação preconizada.

Quando avaliado o nível de atividade física por domínio do IPAQ, verificou-se que mais da metade das mulheres foram insuficientemente ativas ou sedentárias nos domínios trabalho $(64,4 \%)$, deslocamento $(78,1 \%)$, tempo livre $(85,2 \%)$ e atividades domésticas (54,3\%).

Tabela 2 - Caracterização do nível de atividade física global das mulheres e pelos domínios do IPAQ e do tempo gasto sentado. Salvador, Bahia, Brasil - 2016 (N=143)

(continua)

\begin{tabular}{lc}
\hline Nivel de Atividade Física - IPAQ & n (\%) \\
\hline IPAQ Global & $93(65,4)$ \\
Ativo/Muito ativo & $49(34,5)$ \\
Inativo/Sedentário & \\
Domínio Trabalho (n=59*) & $4(6,8)$ \\
Muito ativo & $17(28,8)$ \\
Ativo & $21(35,6)$ \\
Irregular ativo & $17(28,8)$ \\
Sedentário &
\end{tabular}


Tabela 2 - Caracterização do nível de atividade física global das mulheres e pelos domínios do IPAQ e do tempo gasto sentado. Salvador, Bahia, Brasil - 2016 (N=143)

\begin{tabular}{lc}
\hline Nivel de Atividade Física - IPAQ & n (\%) \\
\hline Domínio Deslocamento & - \\
Muito ativo & $31(21,8)$ \\
Ativo & $77(54,2)$ \\
Irregular ativo & $34(23,9)$ \\
Sedentário & \\
Domínio Tempo Livre & $3(2,1)$ \\
Muito ativo & $18(12,7)$ \\
Ativo & $34(23,9)$ \\
Irregular ativo & $87(61,3)$ \\
Sedentário & \\
Domínio Atividades Domésticas & $1(0,7)$ \\
Muito ativo & $64(45,1)$ \\
Ativo & $61(43,0)$ \\
Irregular ativo & $16(11,3)$ \\
Sedentário & \\
\hline
\end{tabular}

Fonte: Elaboração própria.

* Embora 55 mulheres tivessem situação laboral remunerada, somente 46 delas trabalhavam fora do domicílio.

Nota: Sinal convencional utilizado:

- Dado numérico igual a zero não resultante de arredondamento.

\section{Discussão}

Neste estudo, predominaram mulheres com obesidade grau I a III e menor proporção com sobrepeso. Dados do VIGITEL confirmam maior prevalência da obesidade em mulheres e de sobrepeso em homens ${ }^{(2)}$. Todas estavam com circunferência da cintura acima do recomendado e mais de dois terços com razão cintura quadril (RCQ) elevada, conferindo risco aumentado para eventos cardiovasculares. Tendo em vista que a maioria estava em acompanhamento no serviço há mais de 3 anos, a permanência do excesso de peso indica a dificuldade enfrentada no seu controle. Esse problema evidencia o desafio da equipe multidisciplinar no alcance do controle desse complexo fenômeno, sobretudo em mulheres com elevado grau de obesidade, como constatado neste estudo, uma vez que a quantidade de tentativas para perda de peso é diretamente proporcional ao IMC, isto é, quanto maior o IMC, maior a frequência de tentativas ${ }^{(14)}$.

Ao analisar isoladamente os domínios trabalho, deslocamento e tempo livre, maior proporção das mulheres apresentaram-se sedentárias/insuficientemente ativas. No entanto, eram ativas/muito ativas no domínio atividades domésticas, fato que contribuiu para a classificação da maioria delas como ativas/muito ativas no nível global de atividade física $(65,4 \%)$.

A maioria caracterizou-se pela baixa renda familiar mensal e escolaridade inferior ao nível superior e por não terem empregada doméstica, justificando o melhor nível de atividade física no domínio domicílio. Pessoas com nível educacional mais elevado compartilham mais as atividades domésticas e apresentam-se menos ativas nessas tarefas e aquelas com menor nível de escolaridade são mais ativas no domicílio e menos no trabalho. Esses resultados estão em consonância com outro estudo realizado no município de Salvador (BA), no qual também predominaram mulheres, negras, com baixa escolaridade e mais ativas no domicílio em relação ao trabalho e deslocamento ${ }^{(15)}$.

Neste estudo, menos de um terço das mulheres alcançou o nível de atividade física recomendado no domínio deslocamento, apesar da baixa renda ${ }^{(16)}$. Esse fato pode estar associado 
às variáveis não investigadas que influenciam o nível de atividade física no deslocamento, a exemplo de fatores ambientais, como segurança nas vias públicas, disponibilidade de ciclovias, presença de calçadas e distância da residência para o trabalho. Corroboram os achados deste estudo dados da investigação realizada em 100 municípios de 23 estados brasileiros, com 12.402 adultos e 6.624 idosos, mostrando que 66,6\% dos adultos e $73,9 \%$ dos idosos eram insuficientemente ativos no deslocamento ${ }^{(17)}$. Uma vez que a atividade física nesse domínio é considerada uma estratégia para melhorar o nível de atividade física da população em geral ${ }^{(17)}$, países em desenvolvimento têm investido na qualidade de transportes, acessibilidade e segurança ${ }^{(18)}$. O estímulo ao deslocamento ativo pode melhorar o tráfego e está associado à redução total de 11\% no risco cardiovascular, além de ter forte efeito protetor para mulheres ${ }^{(19)}$.

Quanto ao domínio tempo livre e lazer, a maioria das mulheres estudadas era sedentária/ insuficientemente ativa, em consonância com estudo brasileiro com 2.691 adultos, no município de São Paulo. Além disso, o estudo mostrou que raça/cor negra, menor renda familiar e escolaridade, maior faixa etária e presença de obesidade estavam associadas a inatividade física, características prevalentes neste estudo ${ }^{(20)}$. O baixo nível de atividade física no tempo livre pode estar associado à vulnerabilidade social e histórica causadora de desigualdades em diversas variáveis de saúde, inclusive no comportamento em relação à prática de atividade física ${ }^{(15)}$. É válido destacar que indivíduos com maior escolaridade e renda têm mais oportunidades e facilidades para acesso à prática de atividade física no lazer e maior suporte social para assimilar as recomendações e benefícios desse hábito ${ }^{(20)}$.

Somado a isso, o fato de a maioria das mulheres exercer trabalho remunerado por mais de seis horas por dia, ser ativa nas tarefas domésticas e ter dependente sob seus cuidados pode também ter contribuído para menor disponibilidade de tempo e de oportunidades para as atividades físicas no lazer. Esses fatores também estão presentes no estudo realizado com 2.050 adultos no munícipio de São Paulo ${ }^{(21)}$.

Quanto ao gênero, destaca-se que as mulheres, desde a infância, brincam com bonecas e afazeres domésticos, praticam atividade física por questões de saúde, orientação médica ou por estética, além de serem responsáveis pelo trabalho doméstico, enfrentando dupla jornada, o que pode diminuir o nível de atividade física no contexto do lazer ${ }^{(18,22)}$.

Diante do panorama inatividade física na maioria dos domínios do IPAQ, é necessária a criação de medidas direcionadas aos grupos de maior vulnerabilidade, para que possam acumular mais tempo de atividade física nos diversos domínios ${ }^{(19)}$. Nesse sentido, a equipe multiprofissional apresenta fundamental importância para desenvolver ações que estimulem a valorização da modificação de comportamentos. $\mathrm{O}$ (a) enfermeiro(a) pode sugerir estratégias para a superação de barreiras que dificultem a prática de atividade física, uma vez que a obesidade e o sedentarismo são considerados condições multifatoriais que exigem atuação multiprofissional, objetivando a redução desses problemas e levando em conta as particularidades de cada indivíduo e o projeto de cada um no cuidado de $\mathrm{si}^{(23)}$.

Nessa perspectiva, a enfermagem deve estar articulada com redes de apoio, oferecendo suporte necessário para a perda de peso. A educação em saúde junto às mulheres deve potencializar seu conhecimento sobre males associados à obesidade e benefícios da atividade física para reduzi-la. Deve compartilhar conhecimentos e saberes por meio de uma comunicação sensível e efetiva, estimulando a prática da autonomia e autocuidado de forma reflexiva e dinâmica ${ }^{(23)}$.

Os resultados desta pesquisa assinalam a importância de políticas e programas de promoção da saúde, prevenção e redução de agravos por intermédio do incentivo à prática regular de atividade física, assim como projetos compartilhados de saúde entre profissionais de saúde e as mulheres.

Com relação às limitações do estudo, destaca-se o tamanho da amostra e o estudo transversal, em que a exposição e o desfecho são 
coletados em um único momento no tempo, tornando difícil a determinação de relações causais. Entretanto, os estudos transversais consistem em uma ferramenta de grande utilidade para a descrição de características da população, para a identificação de grupos de risco e para a ação e o planejamento em saúde.

\section{Conclusão}

A maioria das mulheres com excesso de peso é ativa no escore global de atividade física, sobretudo em razão de serem ativas no domínio atividade doméstica. Entretanto, quando analisados os demais domínios (lazer, trabalho e deslocamento) de forma individual, maior proporção delas eram sedentárias/insuficientemente ativas. Desse modo, é necessário encontrar estratégias para otimizar a prática de atividade física sobretudo no lazer.

\section{Colaborações:}

1. concepção, projeto, análise e interpretação dos dados: Taise Santos do Nascimento, Fernanda Carneiro Mussi, Cátia Suely Palmeira, Francisco José Godim Pitanga e Melissa Almeida Santos Paim;

2. redação do artigo e revisão crítica relevante do conteúdo intelectual: Taise Santos do Nascimento, Fernanda Carneiro Mussi, Cátia Suely Palmeira e Francisco José Godim Pitanga;

3. aprovação final da versão a ser publicada: Taise Santos do Nascimento, Fernanda Carneiro Mussi e Melissa Almeida Santos Paim.

\section{Referências}

1. Brasil. Ministério da Saúde. Secretaria de Atenção à Saúde. Departamento de Atenção Básica. Estratégias para o cuidado da pessoa com doença crônica: Obesidade [Internet]. Brasília; 2014 [cited 2016 June 20]. Available from: http://bvsms.saude. gov.br/bvs/publicacoes/estrategias_cuidado_ doenca_cronica_obesidade_cab38.pdf

2. Brasil. Ministério da Saúde. Secretaria de Vigilância em Saúde. Vigilância de fatores de risco e proteção para doenças crônicas por inquérito telefônico [Internet]. Brasília; 2015 [cited 2016 June 20]. Available from: https://www.ans.gov.br/ images/stories/Materiais_para_pesquisa/Materiais_ por_assunto/2015_vigitel.pdf

3. Matsudo VKR, Matsudo SMM. Atividade física no tratamento da obesidade. Einstein. 2006;Supl 1:S29-S43.

4. Silva RC, Diniz MFHS, Alvim S, Vidigal PG, Fedeli LMG, Barreto SM. Atividade física e perfil lipídico no estudo longitudinal de saúde do adulto (ELSABrasil). Arq Bras Cardiol. 2016;107(1):10-9.

5. Joshi S, Mooney SJ, Kennedy GJ, Benjamin EO, Ompad D, Undle AGR, et al. Beyond METs: types of physical activity and depression among older adults. Age Ageing [Internet]. 2016 Jan [cited 2016 Apr 15];45:103-9. Available from: https://www. ncbi.nlm.nih.gov/pmc/articles/PMC4711656/

6. Argento RSV. Benefícios da atividade física na saúde e qualidade de vida do idoso [trabalho de conclusão de curso]. Campinas (SP): Universidade Estadual de Campinas; 2010 [cited 2016 Apr 16]. Available from: file://C:/Users/ribei_000/ Downloads/ArgentoRenedeSouzaVianello_TCC. pdf

7. Joseph RP, Ainsworth BE, Keller C, Dodgson JE. Barriers to physical activity among African American women: an integrative review of the literature. Women Health. 2015;55(6):679-99.

8. Welch N, McNaughton SA, Hunter W, Hume C, Crawford D. Is the perception of time pressure a barrier to healthy eating and physical activity among women? Public Health Nutrit [Internet]. 2009 Jul 23 [cited 2015 June 20];12(7):888-95. Available from: https://www.ncbi.nlm.nih.gov/ pubmed/18647424

9. Tribess S, Virtuoso Júnior JS, Oliveira RJ. Atividade física como preditor da ausência de fragilidade em idosos. Rev Assoc Med Bras. 2012;58(3):341-7.

10. Matsudo S, Araujo T, Matsudo V, Andrade D, Andrade E, Oliveira LC, et al. Questionário internacional de atividade física (IPAQ): estudo de validade e reprodutibilidade no Brasil. Rev Bras Ativid Física Saúde [Internet]. 2001 [cited 2015 Aug 20];6(2):5-18. Available from: http://rbafs.org.br/ RBAFS/article/view/931

11. World Heald Organization. Obesity: adultes [Internet]. Geneva; 2018 [cited 2018 Sep 9]. Available from: http://www.who.int/en/news-room/ fact-sheets/detail/obesity-and-overweight 
12. Heyward VH, Stolarczyk LM. Applied body composition assessment. Champaign (IL): Human Kinetics; 1996.

13. Andrade SC. Prevalência de comportamentos voltados à perda de peso e suas associações com índice de massa corporal e autopercepção [dissertação]. [Internet]. Porto Alegre (RS): Faculdade de Medicina, Pontifícia Universidade Católica do Rio Grande do Sul; 2016 [cited 2017 May 15]. Available from: http://tede2.pucrs.br/ tede2/bitstream/tede/6686/2/DIS_SABRINA_ CHAPUIS_DE_ANDRADE_COMPLETO.pdf

14. Pitanga FJG, Lessa I, Barbosa PJB, Barbosa SJO, Costa MC, Lopes AS. Fatores sociodemográficos associados aos diferentes domínios da atividade física em adultos de etnia negra. Rev bras epidemiol. 2014;15(2):363-75.

15. Hardman CM, Barros SSH, Oliveira ESA, Nahas MV, Barros MVG. Inatividade nos deslocamentos para o trabalho e fatores associados em industriários. Saude soc [Internet]. 2013 set [cited 2018 Sep 8];22(3):760-72. Available from: http://www. scielo.br/scielo.php?script=sci_arttext\&pid=\$010412902013000300010\&lng=en

16. Madeira MC, Siqueira FCV, Facchini LA, Silveira DS, Tomasi E, Thumé E, et al. Atividade física no deslocamento em adultos e idosos do Brasil: prevalências e fatores associados. Cad Saúde Pública. 2013 jan;29(1):165-74.

17. Malta DC, Andrade SSA, Santos MAS, Rodrigues GBA, Mielke GI. Tendências dos indicadores de atividade física em adultos: conjunto de capitais do Brasil 2006-2013. Rev Bras Ativ Fís Saúde [Internet]. 2015 mar [cited 2018 Sep 8];21(4):14151. Available from: http://www.scielo.br/pdf/csc/ v21n4/1413-8123-csc-21-04-1061.pdf

18. Mielke GI, Hallal PC, Rodrigues GBA, Szwarcwald CL, Santos FV, Malta DC. Prática de atividade física e hábito de assistir à televisão entre adultos no Brasil: Pesquisa Nacional de Saúde 2013. Epidemiol Serv Saúde. 2015 abr-jun;24(2):277-86.

19. Sousa CA, César CLG, Barros MBA, Carandina L, Goldbaum M, Marchioni DML, et al. Prevalência de atividade física no lazer e fatores associados: estudo de base populacional em São Paulo, Brasil, 2008-2009. Cad Saúde Pública [Internet]. 2013 fev [cited 2018 Sep 8];29(2):270-82. Available from: http://www.scielo.br/scielo.php?script $=$ sci_ arttext\&pid=S0102-311X2013000200014\&lng=en

20. Sá-Silva SP, Yokoo EM, Salles-Costa R. Fatores demográficos e hábitos de vida relacionados com a inatividade física de lazer entre gêneros Rev Nutr. 2013 nov/dez;26(6):633-45.

21. Zanchetta LM, Barros MBA, César CLG, Carandina L, Goldbaum M, Alves MCGP. Inatividade física e fatores associados em adultos, São Paulo, Brasil. Rev bras epidemiol [Internet]. 2010 set [cited 2018 Sep 8];13(3):387-99. Available from: http://www. scielo.br/scielo.php?script $=$ sci_arttext $\&$ pid $=$ S1415790X2010000300003\&lng=en

22. Pires CG, Mussi FC, Cerqueira BB, Pitanga FJ, Silva DO. Prática de atividade física entre estudantes de graduação em enfermagem. Acta Paul Enferm [Internet]. 2013 [cited 2018 Sep 8];26(5):436-43. Available from: http://www. scielo.br/scielo.php?script $=$ sci_arttext $\&$ pid $=$ S010321002013000500006\&lng=en

23. Moura PC, Braga LM, Domingos CS, Rodrigues NV, Correia MDL, Oliveira LVA. Diagnósticos e intervenções de enfermagem em indivíduos hipertensos e diabéticos à luz de Orem. Rev Rene. 2014 dez;15(6):1039-46.

Recebido: 8 de março de 2018 Aprovado: 25 de setembro de 2018 Publicado: 10 de dezembro de 2018

A Revista Baiana de Enfermagem utiliza a Licença Creative Commons - Atribuição-NãoComercial 4.0 Internacional. https://creativecommons.org/licenses/by-nc/4.0/

Este artigo é de acesso aberto distribuído sob os termos da Licença Creative Commons (CC BY-NC). Esta licença permite que outros remixem, adaptem e criem a partir do seu trabalho para fins não comerciais. Embora os novos trabalhos tenham de lhe atribuir o devido crédito e não possam ser usados para fins comerciais, os usuários não têm de licenciar esses trabalhos derivados sob os mesmos termos. 